\title{
Metastatic Uterine Carcinosarcoma: A Case Report and Literature Review
}

\author{
Erica Testani OMSIII' ${ }^{*}$, Michael Smith DO $^{1}$, Richard Hwang MD², Ali Chaudri MD ${ }^{2}$ and Leacroft Green MD ${ }^{1}$ \\ ${ }^{1}$ Department of Surgery, St. Barnabas Hospital, USA \\ ${ }^{2}$ Department of Pathology, St. Barnabas Hospital, USA
}

Submission: February 19, 2017; Published: May 30, 2017

*Corresponding author: Erica Testani OMSIII, Department of Surgery, St. Barnabas Hospital, Bronx, New York, USA, Email: etestani@nyit.edu

Abstract

Uterine carcinosarcoma (UCS) is a highly aggressive malignancy with a poor prognosis. Metastasis of UCS is seen most frequently in the abdomen, pelvis and lungs. This report discusses a rare case of a post-menopausal female with recurrent UCS who was diagnosed with metastasis to the soft tissue of the left anterior neck. In 2011, the patient was initially diagnosed with stage III UCS and successfully treated with a total abdominal hysterectomy, bilateral salpingo-oophorectomy, and regional lymphadenectomy, followed by adjuvant radiation and chemotherapy. The patient was in clinical remission and screening PET scans were negative for a 2.5-year period. In 2015, a PET scan and biopsy revealed metastatic spread of UCS to the anterior neck, pulmonary parenchyma and axillary lymph nodes. The present review discusses UCS, a rare presentation of distant metastasis and the mechanisms underlying cancer dormancy.

\section{Clinical Presentation}

A 56 year old G4P3013 Hispanic female presented to outpatient clinic in November 2011 with a 6 month history of daily postmenopausal bleeding. The patient's medical history included a Papanicolaou test demonstrating atypical squamous cells of unknown significance (ASCUS) within the past year. She denied any medical conditions or history of hormone replacement therapy. She reported menarche at age 12 and menopause at age 46 .

Initial physical exam revealed a firm cervix and a firm anteriorly fixed uterus consistent with 6-8 weeks gestation. Transvaginal ultrasound demonstrated a thickened endometrial stripe of 5.6 centimeters (cm) and an endometrial biopsy revealed moderately differentiated endometrial adenocarcinoma. Subsequent total body CT scan showed several areas of lymphadenopathy, specifically in the left internal iliac nodes and left external iliac nodes and multiple retroperitoneal locations, including the para-aortic lymph nodes. Pelvic scans showed endometrial expansion with a heterogeneous mass measuring $9.3 \times 6.4 \times 10.0 \mathrm{~cm}$.

In February 2012, the patient underwent an exploratory laparotomy, radical total abdominal hysterectomy, bilateral salphingo-oophorectomy and regional lymphadenectomy. Intraoperative findings included invasion into the uterine serosa and cervix with greater than $50 \%$ myometrial invasion. On gross examination the cervix and uterus measured $12.0 \times 9.0 \times 8.0 \mathrm{~cm}$. The cervix showed an irregular papillary growth occupying the entire cervical canal and extension into the lower segment of the uterus.

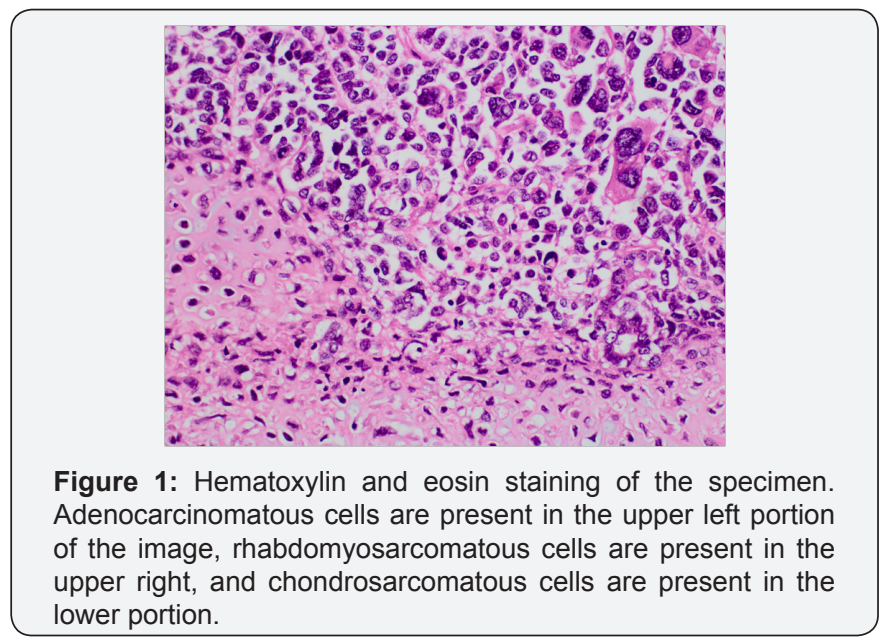

The endometrium was completely occupied by a soft variegated and hemorrhagic tumor mass. Histology of the specimen demonstrated poorly differentiated malignant neoplasm morphologically compatible with metastatic uterine carcinosarcoma (UCS), demonstrating S-100 protein immunohistochemical staining for the chondrosarcomatous 


\section{Juniper Online Journal of Case Studies}

component and myogenin immunohistochemical stain of the rhabdomyosarcomatous component, (Figures 1-3). Only one of eleven lymph nodes, specifically the right obturator lymph node, was positive for metastatic disease. Pelvic washing cytology was negative for malignant cells. TMN staging was determined to be pT2 pN1 pMX, Fédération Internationale de Gynécologie et d'Obstétrique (FIGO) stage IIIC1.

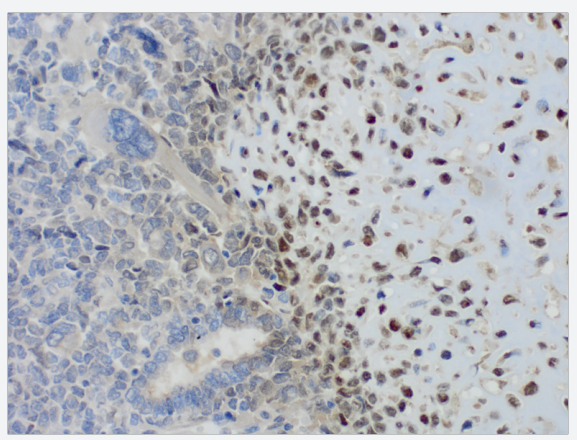

Figure 2: S-100 protein staining demonstrating the chondrosarcomatous cells (right) while the adenocarcinoma and rhabdomyosarcoma components (left) are unstained.

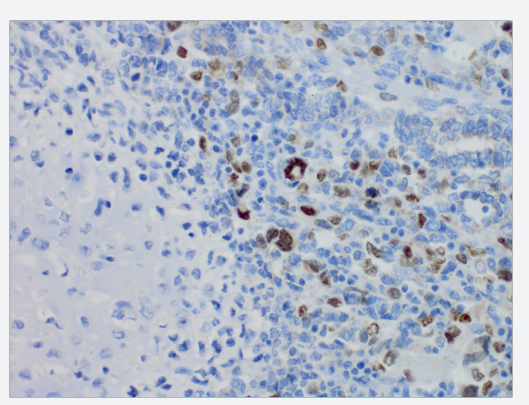

Figure 3: Immunohistochemical staining with myogenin which highlights the rhabdomyosarcomatous component of the tumor.

During an 18-month period following surgical resection, the patient underwent 6 cycles of radiation therapy and chemotherapy including four cycles of taxane/anthracycline/ platinum (TAP) and two cycles of carboplatin/paclitaxel. PET scans in May 2013 confirmed absence of disease with complete remission. Recurrence was monitored with serial CT scans every six months and tumor marker (CA 125, CA 19-9 and CEA) screening every three months.

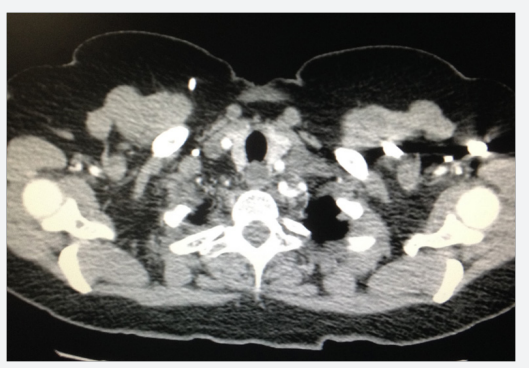

Figure 4: Axial image from CT scan performed in April 2016.

In October 2015 a PET scan revealed pulmonary parenchymal metastasis, left axillary adenopathy and left cervical adenopathy lateral to the thyroid gland. Tumor marker CA 125 was increased to 40 units per milliliter, a significant elevation above the normal threshold $(<35 \mathrm{U} / \mathrm{mL})$. Left supraclavicular lymph node FNA confirmed metastatic recurrence of carcinosarcoma. The patient received 4 cycles of carboplatin/paclitaxel chemotherapy in November 2015. PET scan in February 2016 demonstrated good response to treatment, follow up CT in April 2016 did not reveal any masses, (Figure 4).

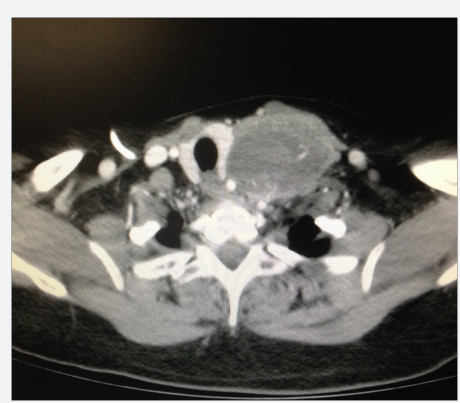

Figure 5: Axial section at same level as figure 1 performed in October 2016.

In October 2016 a CT with contrast demonstrated a new soft tissue mass on the left neck with vascular supply originating from a branch of the subclavian artery, (Figure 5). One month later, the patient underwent a modified neck dissection. The specimen was an $8.5 \times 7.0 \times 4.5 \mathrm{~cm}$ soft tan-yellow mass with areas of hemorrhage and necrosis, (Figure 6 \& 7). Histopathology demonstrated metastatic carcinosarcoma with identical histopathologic features as the previous uterine tumor. Consent was obtained from the patient for intra operative photography and the production of this manuscript was approved by our institution's internal review board.

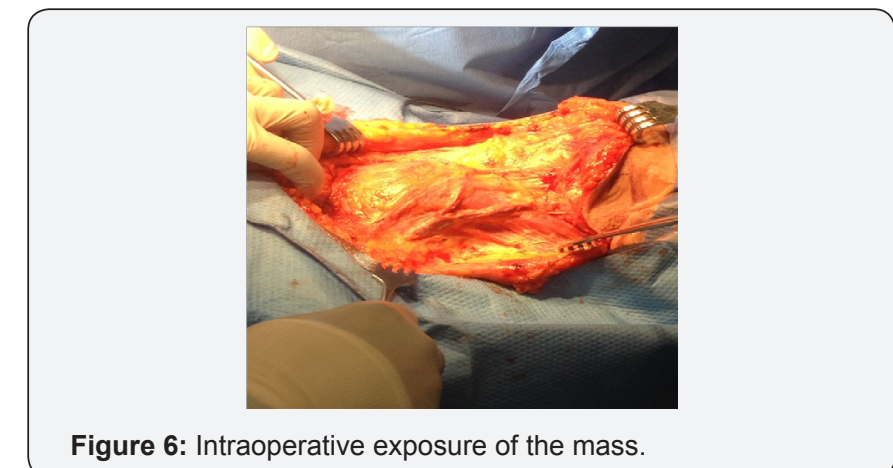

Figure 6: Intraoperative exposure of the mass.

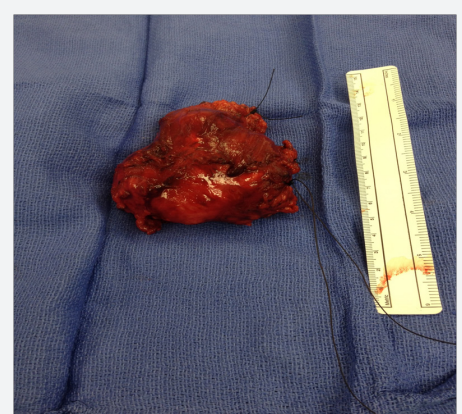

Figure 7: Excised mass: the short suture is the superior margin, the long suture is the lateral margin. 


\section{Literature Review}

Uterine carcinosarcoma (UCS), also known as a malignant, mixed Müllerian tumor (MMMT) is a highly aggressive cancer that accounts for approximately $3 \%$ of all uterine malignancies [1]. The majority of cases are seen in post-menopausal women with a median age of 65 [2]. Fewer than $5 \%$ of cases are diagnosed in women younger than 50 years old. Data from the National Cancer Institute indicates that black females have significantly higher incidence rates of UCS and poorer treatment outcomes compared with white non-Hispanic females [3].

UCS grossly presents as a bulky, polypoid mass with necrotic and hemorrhagic foci. The tumor often fills the endometrial cavity, invades deep into the myometrium and can protrude into the cervix [3]. The most common initial presenting symptom is abnormal postmenopausal uterine bleeding. Additional signs and symptoms include gradual weakness, increasing abdominal girth, pelvic pain and pressure, and a protuberant cervical mass. Risk factors are similar to that of endometrial carcinoma and are associated with an excess estrogen environment as seen in obesity, nulliparity, menstrual irregularities and long-term unopposed estrogen therapy. There is some evidence to suggest that UCS is also associated with previous pelvic irradiation and prolonged Tamoxifen use [4]. Carcinosarcoma is unique in that it is biphasic and contains both malignant epithelial and mesenchymal components [5]. Microscopically the epithelial elements are typically high grade endometrial adenocarcinoma that can be serous, endometrioid, clear cell, mucinous or squamous cell.

The histology of the mesenchymal component can be subdivided into homologous or heterologous types of uterine tissue. Homologous mesenchyme usually resembles endometrial stromal sarcoma or fibrosarcoma. The heterologous subtype is derived from undifferentiated sarcoma and contains components of skeletal muscle, cartilage or bone. The most common variant is rhadbomyosarcoma, followed by chondrosarcoma, osteosarcoma and liposarcoma [1]. The prognostic significance of the mesenchymal histology is controversial however, most studies suggest that the homologous or heterologous elements do not influence the behavior of the tumor [3]. Conversely, tumors with an epithelial component composed of grade III endometroid, serous or clear cell type are associated with a higher incidence of metastasis and deep myometrial and cervical invasion [4].

Clinical and pathological evidence suggests that MMMT derives from an endometrial carcinoma that has undergone a metaplastic change resulting in the acquisition of sarcomatous features [5]. This theory explains why UCS behaves like an aggressive subtype of endometrial carcinoma rather than a uterine sarcoma. Despite its similarities, clinical evidence suggests that UCS is biologically distinct from endometrial carcinoma because of its significantly worse prognosis and potential for hematogenous spread [5]. At the time of diagnosis, approximately $50 \%$ of all cases are FIGO stage III or stage IV and present with either extra uterine spread or distant metastasis.

The pattern of UCS spread is primarily governed by the carcinomatous component of the tumor. Immunohistochemical analysis has demonstrated the majority of metastatic foci contain predominantly carcinomatous elements [6]. Sarcomatous elements can be found in metastatic tumors however purely sarcomatous tissue is uncommon [4]. Therefore, like endometrial carcinoma, MMMT predominantly spreads through the lymphatic system.

The most common sites of metastasis are the lung, peritoneum and pelvic and para-aortic lymph nodes [1]. Forty to sixty percent of women will experience disease recurrence, which often presents in the abdomen, pelvis, vagina, and lungs [7]. In most cases the median survival is approximately 21 months and overall 5 year survival ranges from 33-39\% [8].

Surgery is the primary treatment for uterine carcinosarcoma and includes total abdominal hysterectomy, bilateral salpingoophrectomy, lymph node dissection, omentectomy or omental biopsy and resection of all gross disease. Several studies demonstrate that lymphadenectomy confers a measurable survival benefit by removing microscopic lymphatic metastasis.

Therefore, research suggests that the number of lymph nodes resected is an important predictor of prognosis and survival [9]. Other prognostic factors include degree of lymphovascular space involvement, depth of myometrial invasion, adnexal and serosal involvement, lymph node metastasis and positive peritoneal cytology [7]. Regardless of the aforementioned factors or stage, adjuvant chemotherapy or radiation therapy is recommended in all women because of the high rate of disease recurrence [3].

\section{Discussion}

This case demonstrates a clinical phenomenon known as cancer dormancy, in which a patient experiences a period of asymptomatic remission after resection of the primary tumor. Latent cancer cells persist below a detectable threshold for months to decades until the disease eventually recurs, often in the form of metastatic relapse. Dormancy is well documented in cases of recurrent breast, prostate, thyroid, brain and pancreatic cancers [10]. Research shows that disseminated tumor cells (DTCs) have been detected in asymptomatic patients with a history of breast cancer, even decades after removal of the primary tumor. Some studies suggest that many patients who are considered cured still house latent cancer cells [11].

The precise mechanism underlying cell dormancy is unknown. It is likely due to a combination of mutations in genes modulating cell proliferation and alterations in the surrounding microenvironment. There are three prevailing models for understanding this phenomenon: angiogenic dormancy, immunologic dormancy and cellular dormancy [12]. 
Angiogenic dormancy occurs when the proliferation of malignant cells is restricted by a finite blood supply. An enlarging tumor mass must rely on neo vascularization to provide sufficient nutrients and oxygen to sustain growth [13]. Without an increasing vascular supply, DTCs will exist in a state of equilibrium in which rapid proliferation is balanced by a comparable rate of cell death. Malignant cells escape dormancy when genetic alterations initiate an angiogenic shift which results in the up regulation of proangiogenic factors, such as VEGF, which restores the potential for tumor growth [12]. Research has shown that augmented VEGF expression correlates with rapid induction of tumor growth, while withdrawal of VEGF results in the regression of tumor vessels and extensive cell necrosis $[14,15]$.

Immunologic dormancy occurs when the immune system eradicates or impedes the growth of malignant cells. Immunosurveillance mechanisms detect transformed cancer cells via distinct inflammatory signals and tumor-specific markers. The presence of malignant cells will activate the innate and adaptive immune systems and initiate a cascade of events which ultimately suppress tumor growth [16]. T cells are critical to the induction of cancer dormancy.

$\mathrm{T}$ cells release cytokines, such as INF- $\gamma$ and TNF- $\alpha$, which inhibit cell growth, prevent angiogenesis, and regulate cell cycle progression. In a mouse model of sarcoma, depletion of CD8+ and CD4+ T cells resulted in the growth of previously dormant cancer cells and decreased the overall time to metastasis $[17,18]$.

The immune system is capable of inducing a state of equilibrium between healthy cells and tumor cells. In an immunocompetent host, immune cells will monitor tumor mass and restrict malignant proliferation using a combination of cytostatic and cytolytic factors [19]. Tumor mass will remain constant and the patient will be clinically asymptomatic $[13,16]$. Over time cancer cells can adapt to the immune environment and accumulate genetic mutations that promote immune evasion, neovascularization and proliferation. Ultimately these changes will enable DTCs to escape dormancy resulting in metastatic relapse [10].

Cellular dormancy is a quiescent state in which tumor cells are arrested in the G0 phase of the cell cycle. Latent cells remain in distant tissues and neither divides nor undergoes apoptosis. Cell cycle arrest is driven by a number of intrinsic and extrinsic factors [19]. For example, changes in the tumor microenvironment, such as hypoxia, can prompt cancer cell latency. Radiation therapy can also induce cellular quiescence via the upregulation of cyclin dependent kinase (CDK) inhibitors, which prevent cell cycle progression at the G1 and S phase [16]. Under certain circumstances, such as changes in growth factors, cytokines or nutrients, dormant cells can reenter the cell cycle and resume proliferation [19].
Tumor dormancy presents a number of obstacles to cancer treatment. Latent tumor cells are clinically silent and are resistant to conventional therapy, which primarily targets actively dividing cells. Current research is exploring the use of novel therapeutics designed to shift cells in and out of dormancy. The goal is to encourage DTCs to reenter the cell cycle in order to increase sensitivity to chemotherapeutic agents [12]. The behavior of dormant cancer cells can be highly divergent from that of the primary tumor.

Tumor cells that disseminate early during the course of the disease may eventually evolve and develop different characteristics compared to the primary tumor. Recent evidence shows that DTCs have different genetic variations compared with primary tumor cells [13]. Therefore, metastatic cancers may be resistant to treatments which were designed exclusively for the primary tumor [17].

Dormant DTCs are believed to be the cause of recurrence and metastasis. There are still many unknowns regarding the mechanism of dormancy. Understanding this process is crucial to preventing metastatic relapse. Future research must explore ways of identifying DTCs and eliminating them before they escape dormancy and cause disease recurrence.

Our patient is currently undergoing palliative treatment with radiation and chemotherapy. Despite aggressive treatment with carbo/taxol, weekly gemcitabine and radiation therapy, imaging studies reveal persistent and increasing metabolic uptake in the left neck although no new masses have been identified to date.

\section{References}

1. Froio E, D’Adda T, Fellegara G, Martella E, Caruana P, et al. (2009) Uterine carcinosarcoma metastatic to the lung as large-cell neuroendocrine carcinoma with synchronous sarcoid granulomatosis. Lung Cancer 64(3): 371-377.

2. Galaal K, Kew FM, Lopes A, Meirovitz M, Naik R, et al. (2009) Evaluation of prognostic factors and treatment outcomes in uterine carcinosarcoma. European Journal of Obstetrics and Gynecology and Reproductive Biology 143: 88-92.

3. Menczer J (2015) Review of recommended treatment of uterine carcinosarcoma. Curr Treat Options Oncol 16(11): 53-71.

4. McCluggage WG (2002) Uterine carcinosarcomas (malignant mixed mullerian tumors) are metaplastic carcinomas. International Journal of Gynecologic Cancer 12: 687-690.

5. Bansal N, Herzog TJ, Seshan VE, Schiff PB, Burke WM, et al. (2008) Uterine carcinosarcomas and grade 3 endometrioid cancers: Evidence for distinct tumor behavior. Obstet Gynecol 112(1): 64-70.

6. Singh R (2014) Review literature on uterine carcinosarcoma. Journal of Cancer Research and Therapeutics 10(3): 461-468.

7. Harano K, Hirakawa A, Yunokawa M, Nakamura T, Satoh T, et al. (2016) Prognostic factors in patients with uterine carcinosarcoma: a multiinstitutional retrospective study from the Japanese Gynecologic Oncology Group. International Journal of Clinical Oncology 21: 168176. 
8. Vorgias G, Fotiou S (2010) The role of lyphadenectomy in uterine carcinosarcomas: a critical literature review. Arch Gynecol Obstet 282(6): 659-664.

9. Temkin SM, Hellmann M, Lee YC, Abulafia O (2007) Early-stage carcinosarcoma of the uterus: the significance of lymph node count. Int J Gynecol Cancer 17: 215-219.

10. Udagawa $\mathrm{T}$ (2008) Tumor dormancy of primary and secondary cancers. APMIS 116(7-8): 615-628.

11. Kang Y, Pantel K (2013) Tumor cell dissemination: emerging biological insights from animal models and cancer patients. Cancer Cell 23(5): 573-581.

12. Yeh AC, Ramaswamy S (2015) Mechanisms of cancer cell dormancy Another hallmark of Cancer? Cancer Research 75(23): 5014-5022.

13. Paez D, Labonte MJ, Bohanes P, Zhang W, Benhanim L, et al. (2011) Cancer dormancy: A model of early dissemination and late cancer recurrence. Clin Cancer Res 18(3): 645-653.

14. Benjamin LE, Keshet E (1997) Conditional switching of vascular endothelial growth factor (VEGF) expression in tumors: Induction of endothelial cell shedding and regression of hemangioblastoma-like vessels by VEGF withdrawal. Proc Natl Acad Sci USA 94: 8761-8766.

15. Indraccolo S, Stievano L, Minuzzo S, Tosello V, Esposito G, et al. (2006) Interruption of tumor dormancy by a transient angiogenic burst within the tumor microenvironment. Proceedings of the National Academy of Sciences 103(11): 4216-4221.

16. Evans EB, Lin SY (2015) New insights into tumor dormancy: Targeting DNA repair pathways. World J Clin Oncol 6(5): 80-88.

17. Sosa MS, Bragado P, Aguirre Ghiso J (2014) Mechanisms of disseminated cancer cell dormancy: an awakening field. Nat Rev Cancer 14(9): 611622.

18. Koebel CM, Vermi W, Swann JB, Zerafa N, Rodig SJ, et al. (2007) Adaptive immunity maintains occult cancer in an equilibrium state. Nature 450(6): 903-908.

19. Senft D, Ronai ZA (2015) Immunogenic, cellular, and angiogenic drivers of tumor dormancy - a melanoma view. Pigment Cell Melanoma Res 29(1): 27-42.

\section{Your next submission with Juniper Publishers will reach you the below assets}

- Quality Editorial service

- Swift Peer Review

- Reprints availability

- E-prints Service

- Manuscript Podcast for convenient understanding

- Global attainment for your research

- Manuscript accessibility in different formats

( Pdf, E-pub, Full Text, Audio)

- Unceasing customer service

Track the below URL for one-step submission https://juniperpublishers.com/online-submission.php 